\title{
A Capacitor-Free, Fast Transient Response Linear Voltage Regulator In a 180nm CMOS
}

Deleuran, Alexander N.; Lindbjerg, Nicklas; Pedersen, Martin K. ; Llimos Muntal, Pere; Jørgensen, Ivan Harald Holger

\section{Published in:}

Proceedings of NORCAS 2015

Link to article, DOI:

10.1109/NORCHIP.2015.7364358

Publication date:

2015

Document Version

Peer reviewed version

Link back to DTU Orbit

Citation (APA):

Deleuran, A. N., Lindbjerg, N., Pedersen, M. K., Llimos Muntal, P., \& Jørgensen, I. H. H. (2015). A CapacitorFree, Fast Transient Response Linear Voltage Regulator In a 180nm CMOS. In Proceedings of NORCAS 2015 IEEE. https://doi.org/10.1109/NORCHIP.2015.7364358

\section{General rights}

Copyright and moral rights for the publications made accessible in the public portal are retained by the authors and/or other copyright owners and it is a condition of accessing publications that users recognise and abide by the legal requirements associated with these rights.

- Users may download and print one copy of any publication from the public portal for the purpose of private study or research.

- You may not further distribute the material or use it for any profit-making activity or commercial gain

- You may freely distribute the URL identifying the publication in the public portal 


\title{
A Capacitor-Free, Fast Transient Response Linear Voltage Regulator In a 180nm CMOS
}

\author{
Alexander N. Deleuran, Nicklas Lindbjerg, Martin K. Pedersen, Pere Llimós Muntal and Ivan H.H. Jørgensen \\ Department of Electrical Engineering \\ Technical University of Denmark, Kgs. Lyngby, Denmark \\ s130382@student.dtu.dk,s130381@student.dtu.dk, s125187@student.dtu.dk,plmu@elektro.dtu.dk, ihhj@elektro.dtu.dk
}

\begin{abstract}
A $1.8 \mathrm{~V}$ capacitor-free linear regulator with fast transient response based on a new topology with a fast and slow regulation loop is presented. The design has been laid out and simulated in a $0.18 \mu \mathrm{m}$ CMOS process. The design has a low component count and is tailored for system-on-chip integration. A current step load from $0-50 \mathrm{~mA}$ with a rise time of $1 \mu$ s results in an undershoot in the output voltage of $140 \mathrm{mV}$ for a period of $39 \mathrm{~ns}$. The regulator sources up to $50 \mathrm{~mA}$ current load.
\end{abstract}

\section{INTRODUCTION}

In contemporary low power CMOS integrated circuits, multiple supply voltages are often a necessity for optimizing chip area and power efficiency. Linear regulators excel at providing low output noise and less electromagnetic emission compared to switching mode regulators. Opposed to switching regulators, linear regulators do not require external inductors and are generally less space consuming. Despite a lower power efficiency, linear regulators can be designed to draw a noticeably low quiescent current, i.e. the sum of bias currents during unloaded conditions, since these designs do not depend on a minimum duty cycle. This is advantageous for handheld systems where most energy is consumed in stand-by mode [1].

Due to a finite bandwidth of linear regulators, conventional designs require a high value buffer capacitor, frequently situated off-chip [2]. In portable devices with strict requirements on space consumption, such as hearing aids or cell phones, usage of discrete components must be minimized. This has lead to the development of numerous capacitor-free regulator topologies [3]-[5]. The external capacitor ensures stability and acts as a supply for the frequency components of the current load, $I_{L}$, outside the bandwidth of the regulator. With fast changing current loads an exclusion of the capacitor will lead to large voltage drops on the output and a longer duration of transient recovery, i.e. rise time, $T_{R}$.

One approach of avoiding this large capacitor is by emulating the capacitance using an internal operational amplifierbased active circuit as done in [4]. However, the design is rather complex and utilizes a low dropout methodology with a PMOS pass transistor. Considering the lower charge carrier mobility in most PMOS devices, more area is consumed compared to an NMOS with the same drain current. This increases the gate capacitance and leads to a longer $T_{R}$. Another approach is to increase the bandwidth of the control loop to a level where the regulator is able to compensate for the fast changing current loads [3]. This is achieved by controlling the pass transistor with a simple single stage error

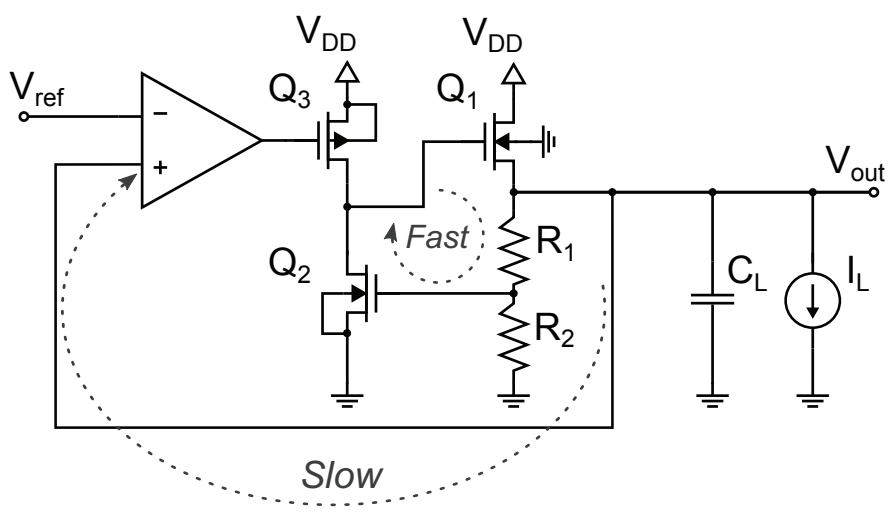

Fig. 1: Functional diagram of the proposed linear voltage regulator

amplifier. In [3] the transient performance is enhanced by an assisting amplifier and the DC output level is stabilized by a low bandwidth amplifier in a parallel control loop.

The new design proposed in this work is based on a principle similar to [3], employing two control loops and an NMOS pass transistor configured as a source follower (SF). Refer to Fig. 1 for the circuit diagram of the proposed regulator. The design specifications target the following parameters. The regulator is supplied by voltage of $3.3 \mathrm{~V}$ and an outputs a voltage of $1.8 \mathrm{~V}$. The regulator can source an $I_{L}$ of $0-50 \mathrm{~mA}$ which can be stepped with a $1 \mu$ s rise -and fall time. The output voltage undershoots less than $200 \mathrm{mV}$ during current step load and the circuit consumes less than $100 \mu \mathrm{A}$ without load. A $C_{L}$ of $1 \mathrm{pF}$ or less will not cause ripple on the output. The design is intended for small products like hearing aids. All transistors in the circuit are $5 \mathrm{~V}$ MOSFETs.

\section{CIRCUIT DESCRIPTION}

The fast loop consists of a common source (CS) amplifier, $\mathrm{Q}_{2}$ and $\mathrm{Q}_{3}$, driving the pass transistor $\mathrm{Q}_{1}$. The current source $\mathrm{Q}_{3}$ is controlled by the slow loop comprising the operational amplifier. The proposed design does not contain any large passive devices and has a low count of transistors. Consequently the simplicity allows for easy and space efficient implementation, yet demonstrating good performance. $C_{L}$ depicts the load capacitance The following sections describe the two control loops in detail. A full circuit diagram is depicted on Fig. 2. 


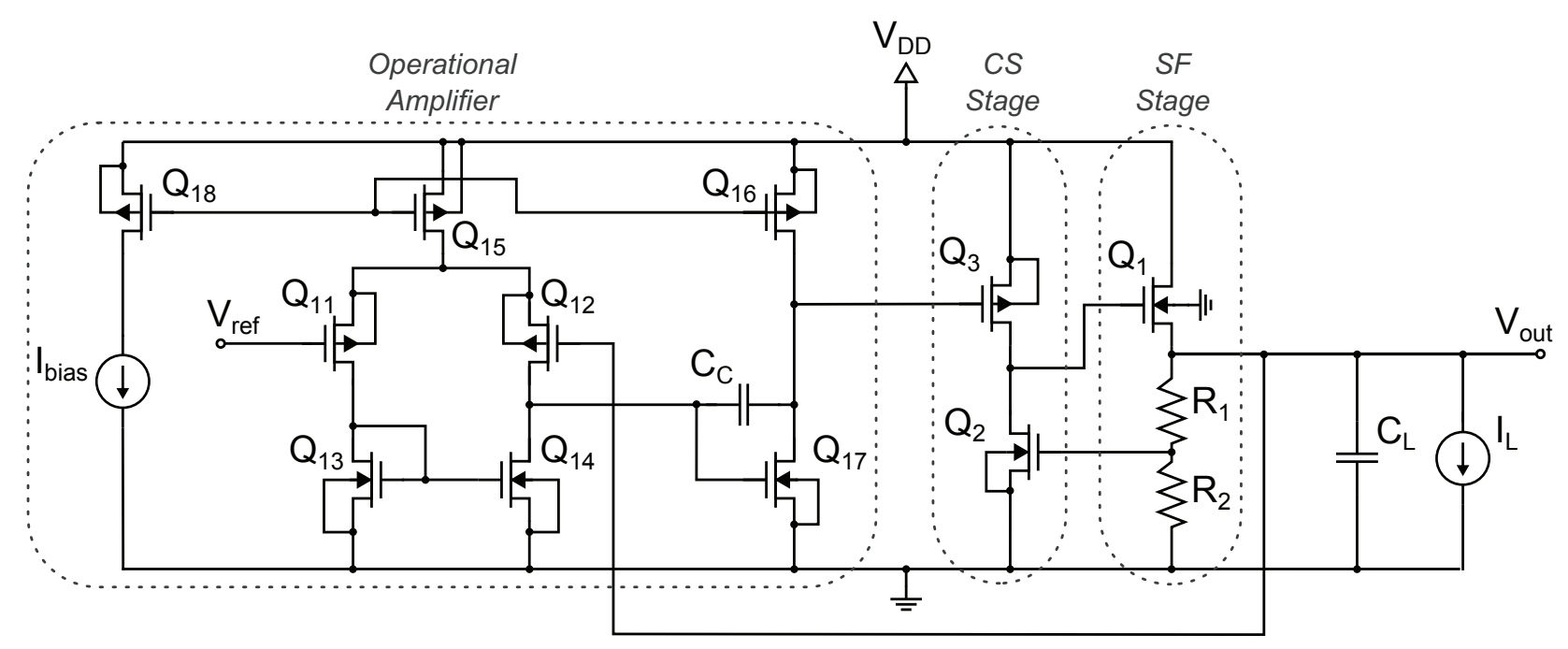

Fig. 2: Full schematic of the proposed linear regulator

\section{A. Fast Loop}

By assuming the fast loop constitutes an underdamped system, the gain bandwidth product (GBWP) of the open loop gain will be inversely proportional to $T_{R}$. The open loop starts at the gate of $\mathrm{Q}_{2}$ and ends at the source of $\mathrm{Q}_{1}$ Based on the former assumption, an uncompensated error amplifier with a maximized $G B W P / I_{D}$ can be employed in order to exploit most of the quiescent current for control speed. $I_{D}$ is the drain current, here spent in the gain stage of the amplifier.

$$
\begin{gathered}
A_{O L}(s)=\left(\frac{g_{m 2} R_{c s} R_{L}^{\prime}}{R_{L}^{\prime}+1 / g_{m 1}}\right) \frac{\left(1+\frac{s}{\omega_{z}}\right)}{\left(1+\frac{s}{\omega_{p 1}}\right)\left(1+\frac{s}{\omega_{p 2}}\right)} \\
\text { where } R_{L}^{\prime}=\left(R_{1}+R_{2}\right)\left\|r_{d s 1}\right\|\left(1 / g_{s 1}\right) \\
\omega_{p 1}=\frac{\left(1 / R_{c s}\right)\left(g_{m 1}+1 / R_{L}^{\prime}\right)}{C_{L}^{\prime} / R_{c s}+C_{1}\left(g_{m 1}+1 / R_{c s}\right)+C_{g s 1} / R_{L}^{\prime}} \\
\omega_{p 2}=\frac{C_{L}^{\prime} / R_{c s}+C_{1}\left(g_{m 1}+1 / R_{c s}\right)+C_{g s 1} / R_{L}^{\prime}}{C_{g s 1} C_{L}^{\prime}+C_{1}\left(C_{g s 1}+C_{L}^{\prime}\right)} \\
\omega_{z}=g_{m 1} / C_{g s 1}
\end{gathered}
$$

The open loop transfer function, $A_{O L}(s)$, is described in (1) where $R_{c s}$ is the output resistance of the CS stage, $C_{L}^{\prime}$ is $C_{L}$ plus the source-bulk capacitance of $\mathrm{Q}_{1}, C_{g s 1}$ is the gate-source capacitance and $C_{1}$ is the gate-bulk and gate-drain capacitance of $\mathrm{Q}_{1} \cdot r_{d s 1}$ is the output resistance and $g_{s 1}$ is the body transconductance of $\mathrm{Q}_{1}$.

Since the CS stage delivers the gain of fast loop the transconductance of $\mathrm{Q}_{2}, g_{m 2}$, must be maximized to achieve the greatest GBWP. Correspondingly $R_{1}$ and $R_{2}$ are used to decrease the gate voltage of $\mathrm{Q}_{2}$ and thereby drive it into moderate inversion for a higher $g_{m}$. These resistors also bias $\mathrm{Q}_{1}$. The optimum current distribution in the CS and SF, that resulted in the shortest $T_{R}$, was found empirically. The gatesource voltage of $\mathrm{Q}_{1}, V_{g s 1}$, becomes considerably large at maximum $I_{L}$. The body effect additionally increases $V_{g s 1}$, so $\mathrm{Q}_{1}$ must have a very high $W / L$ to keep $\mathrm{Q}_{3}$ in saturation. This vast device area introduces substantial parasitic capacitances in $\mathrm{Q}_{1}$ which will dominate the frequency response of the fast loop in terms of $C_{1}$ and $C_{g s 1}$. To minimize $T_{R}$, the dimensions of $\mathrm{Q}_{1}$ must therefore be kept as low as the effective voltage, $V_{e f f}$, of $\mathrm{Q}_{3}$ allows it. At maximum $I_{L}$ the drainsource voltage of $\mathrm{Q}_{3}$ will be at its minimum and will be the limiting factor when choosing the supply voltage. However, if a slightly lower voltage domain is available, it can be connected to the drain of $\mathrm{Q}_{1}$. In that way the power dissipated in $\mathrm{Q}_{1}$ can be significantly reduced without sacrificing performance. Another limiting factor is the load capacitance. As seen in (2) and (3), greater values of $C_{L}$ will push the poles down in frequency and potentially closer together, and therefore at some point compromise the system stability. This significantly determines the maximum amount of devices that the linear regulator can supply.

The loop gain of the fast loop is defined as $L(s)=$ $A_{O L}(s) \frac{R_{2}}{R_{1}}$. When current step loads are applied, ringing can occur on the output of the regulator due to insufficient phase margin of the loop response. Therefore it is desirable to keep the phase margin of $L(s)$ above 75 degrees at maximum expected load capacitance.

\section{B. Slow Loop}

The role of the slow loop is to control the gate voltage of $\mathrm{Q}_{3}$ and thereby stabilize the DC level at $\mathrm{V}_{\text {out }}$. The well known Miller compensated, two stage operational amplifier (opamp) has been utilized for this function. Transistor $\mathrm{Q}_{11}$ to $\mathrm{Q}_{18}$ and $C_{C}$ constitute the opamp. The slow loop starts at the gate of $\mathrm{Q}_{12}$, then goes through the opamp, from gate to the source of $\mathrm{Q}_{3}$ and then from the gate to the source of $\mathrm{Q}_{1}$.

In order not to degrade the frequency response of the fast loop, this opamp has a unity gain frequency approximately two decades below the one of the fast loop; wherefore the opamp does only require a minimal bias current. When greater 


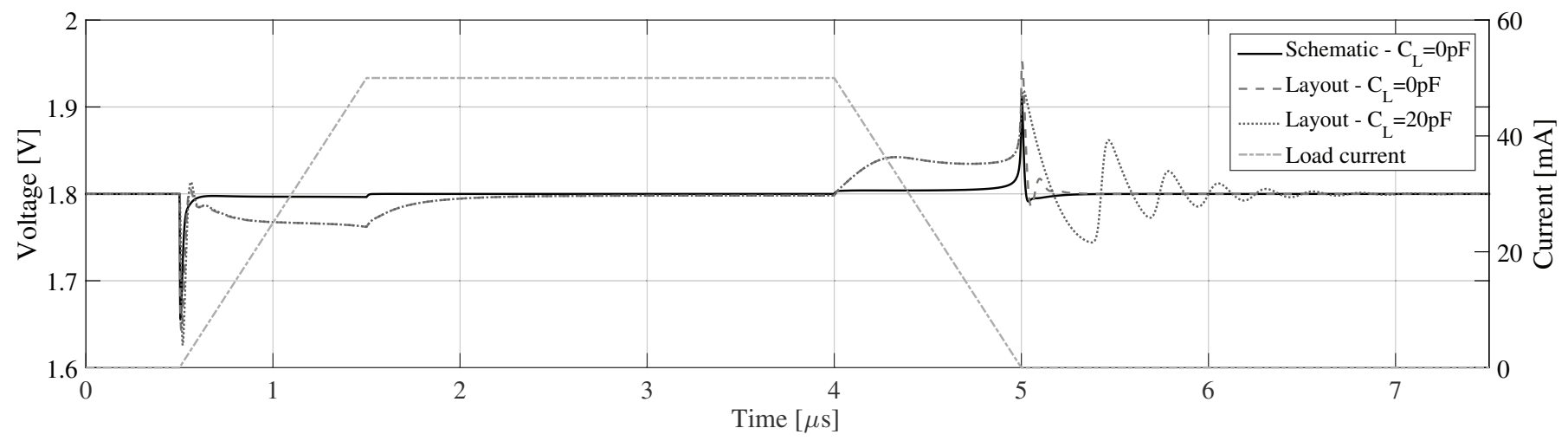

Fig. 4: Transient response with closed slow and fast loop, simulation with and without extracted parasitics

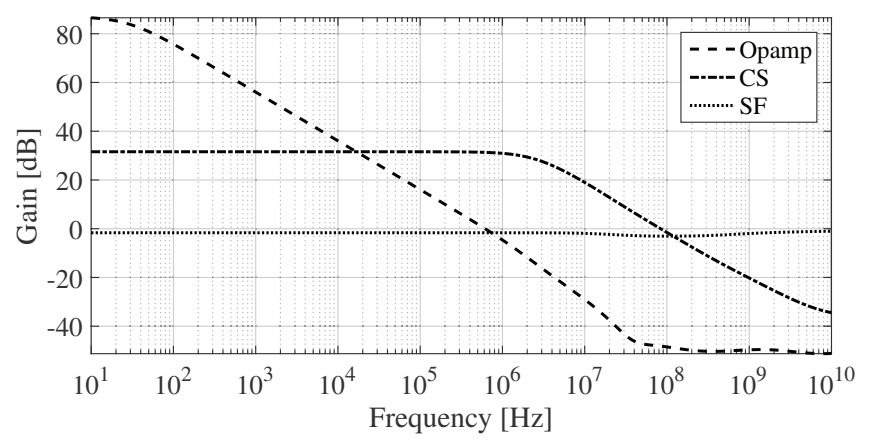

(a) Frequency response of the operational amplifier, the common source stage $\left(\mathrm{Q}_{3}\right.$ isolated from the opamp) and the source follower stage, all without extracted parasitics. $C_{L}=0$ and $I_{L}=0$

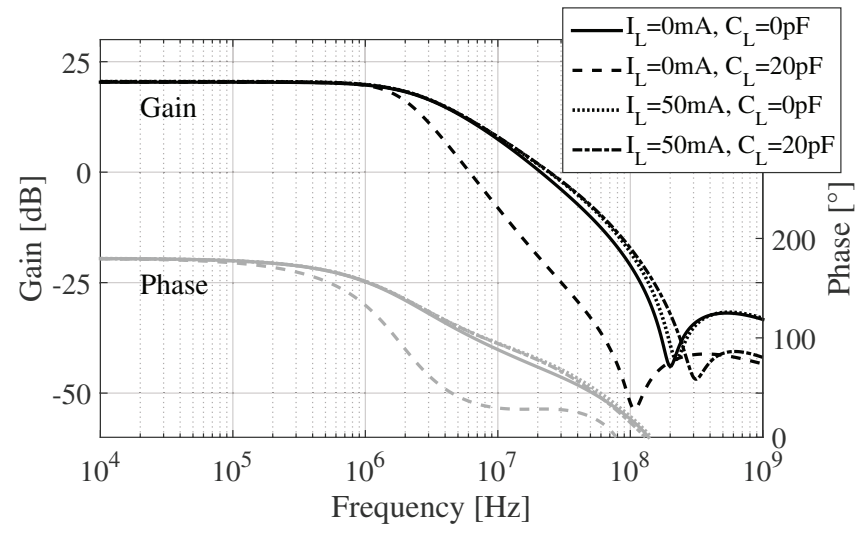

(b) Transfer function of $\mathrm{L}(\mathrm{s})\left(\mathrm{Q}_{3}\right.$ isolated from the opamp), with and without capacitive and current load, all without extracted parasitics

Fig. 3: Simulation results of the proposed linear regulator steps in $I_{L}$ occur the opamp must be able to drive the gate of $\mathrm{Q}_{3}$ without slewing the transient. Therefore, the common source stage of the opamp must provide a sufficiently large drain current of $\mathrm{Q}_{16}, I_{D 16}$. The required $I_{D 16}$ can be reduced by choosing a lower $W / L$ for $\mathrm{Q}_{3}$ to reduce the parasitic capacitance related to the gate. A shorter channel length of $\mathrm{Q}_{3}$ will reduce $R_{c s}$ and thereby decrease $\omega_{p 1}$ which will lead to a lower GBWP. Chosing $W_{3} / L_{3}$ is consequently a compromise between GBWP of the CS stage, $V_{g s}$ of $\mathrm{Q}_{3}$, which also dictates $W_{1} / L_{1}$, and finally the necessary $I_{D 16}$ to reduce slewing.

The design compromises of the slow and fast loop discussed above lead to the device dimensions and drain currents presented in Table I. A value of $300 \mathrm{fF}$ was chosen for $C_{C}$.

\section{Simulation Results}

The proposed capacitor-free linear voltage regulator has been implemented on schematic and layout level in a 0.18 $\mu \mathrm{m}$ CMOS process. The presented results are from the typical temperature and process corner. The most advantageous bias current distribution has been fine tuned by simulation to yield the fastest $T_{R}$. As a result, $82.2 \mu \mathrm{A}$ is distributed to the CS stage, $10 \mu \mathrm{A}$ to the $\mathrm{SF}$ stage and $5.8 \mu \mathrm{A}$ to the opamp, giving a total quiescent current consumption of $98.4 \mu \mathrm{A}$.

The layout is presented in Fig. 4 and has been designed for optimized chip area and measures $150 \mu \mathrm{m} \times 42 \mu \mathrm{m}$. Common centroid matching and dummy devices have been used where necessary and possible. Due to the extremely low $W / L$ of the devices in the opamp, it has not been possible to use unit transistors in the design. The enormous transistor in the left part is $\mathrm{Q}_{1}$ with dimensions $3000 \mu \mathrm{m} / 0.7 \mu \mathrm{m}$.

TABLE I: Device dimensions and drain current

\begin{tabular}{|l|l|l|l|}
\hline Device & Width $[\boldsymbol{\mu m}]$ & Length $[\boldsymbol{\mu m}]$ & $\boldsymbol{I}_{\text {quiescent }}[\boldsymbol{\mu A}]$ \\
\hline $\mathrm{Q}_{1}$ & 3000 & 0.7 & 10 \\
\hline $\mathrm{Q}_{2}$ & 84 & 0.7 & 82.2 \\
\hline $\mathrm{Q}_{3}$ & 140 & 0.6 & 82.2 \\
\hline $\mathrm{Q}_{11,12}$ & 1 & 1 & 0.075 \\
\hline $\mathrm{Q}_{13,14}$ & 0.5 & 4 & 0.075 \\
\hline $\mathrm{Q}_{15}$ & 0.5 & 1 & 0.15 \\
\hline $\mathrm{Q}_{16}$ & 30 & 1 & 5.5 \\
\hline $\mathrm{Q}_{17}$ & 5 & 1 & 5.5 \\
\hline $\mathrm{Q}_{18}$ & 0.5 & 1 & 0.15 \\
\hline
\end{tabular}




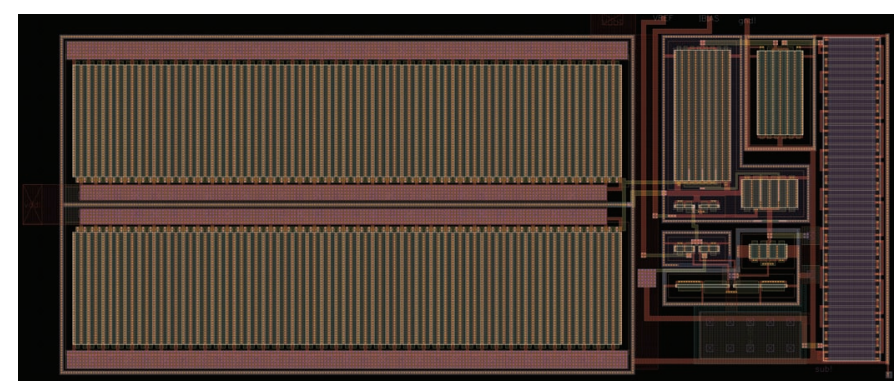

Fig. 4: Screenshot of the layout of the proposed linear regulator

Post-layout simulation has been performed to account for parasitic components in the layout. The frequency responses of the individual circuit segments and the closed loop gain are depicted on Figs. 3a and 3b. It appears that loading the linear regulator by $20 \mathrm{pF}$ will result in an underdamped response due to a phase margin of around 30 degrees. A transient analysis has been performed on schematic and the post-simulated layout level. The circuit was tested with a current step load of $0-50 \mathrm{~mA}$ with a rise -and fall time of $1 \mu \mathrm{s}$. The transient performance is showed on Fig. 4. When simulating with the extracted parasitics, the transient response exhibit a larger and longer voltage drop during transitions in the current step load. This drop might be caused by the capacitance between the metal layers and poly covering the large drain-source and gate area of $\mathrm{Q}_{1}$ respectively. It should be noted that the size of the current step represents a worst case scenario. Under typical circumstances smaller load steps are expected. When a $20 \mathrm{pF}$ load is applied, oscillations occur during step down of $I_{L}$. Referring to Fig. 3b, this response is expected due to the low phase margin. The oscillations only occur during load stepdown because $g_{m 1}$ decreases with the current in $\mathrm{Q}_{1}$ and thereby moves $\omega_{p 2}$ down in frequency according to (3). A higher immunity to $C_{L}$ is conclusively obtained with a greater $g_{m 1}$. A $T_{R}$ of $39 \mathrm{~ns}$ is obtained from the schematic level simulations. When simulating with the extracted parasitics included $T_{R}$ increases to $1.158 \mu \mathrm{s}$. This is a significant difference that indicates layout improvements could better the performance. The voltage undershoot is $140 \mathrm{mV}$ for the schematic and $160 \mathrm{mV}$ for the layout. If the duration of the load step is reduced to $10 \mathrm{~ns}$, a $T_{R}$ of $20.4 \mathrm{~ns}$ is obtained with a $640 \mathrm{mV}$ undershoot on schematic level. Simulations showed that rise times of the load step greater than $1 \mu$ s would result in even lower undershoot voltages.

\section{DISCUSSION}

The presented theory and results of the proposed linear voltage regulator show that an bulky external capacitor can be replaced by a fast control loop. Due to the sensitivity to larger load capacitances, the regulator should supply internal circuitry only. The chip area of the proposed design is fairly small when comparing to the other designs in Table II. Also the design is simple to implement, which makes it ideal for a system-onchip designs. The simulation results from the schematic level and extracted layout simulations of the proposed design are summarized in Table II for comparison with other designs. A figure of merit (FOM) from [1] is used for standardized comparison and appears in (5). As seen, (5) focuses on how
TABLE II: Comparison with other designs

\begin{tabular}{|c|c|c|c|c|c|}
\hline & \multirow{2}{*}{$\mathbf{1 ]}$} & \multirow{2}{*}[\mathbf{4}]{} & \multirow{2}{*}[\mathbf{3}]{} & \multicolumn{2}{|c|}{ This work } \\
\cline { 4 - 6 } & & - & $0.08 \mathrm{~mm}^{2}$ & - & $0.0093 \mathrm{~mm}^{2}$ \\
\hline Active area & $0.0040 \mathrm{~mm}^{2}$ & - & $-8 \mathrm{~V} / 3.6 \mathrm{~V}$ & $3.3 \mathrm{~V}$ & $3.3 \mathrm{~V}$ \\
\hline Supply & $1.2 \mathrm{~V}$ & - & $1.2 \mathrm{~V}$ & $1.8 \mathrm{~V}$ & $1.8 \mathrm{~V}$ \\
\hline Output & $0.9 \mathrm{~V}$ & $2.5 \mathrm{~V}$ & $132 \mathrm{uA}$ & $98.4 \mathrm{uA}$ & $98.3 \mathrm{uA}$ \\
\hline $\boldsymbol{I}_{\text {quiescent }}$ & $6 \mathrm{~mA}$ & $80 \mathrm{uA}$ & $132 \mathrm{~mA}$ & $50 \mathrm{~mA}$ \\
\hline Imax & $100 \mathrm{~mA}$ & $100 \mathrm{~mA}$ & $200 \mathrm{~mA}$ & $50 \mathrm{~mA}$ & $1 \mathrm{us}$ \\
\hline $\boldsymbol{I}_{\boldsymbol{L}}$ Rise time & $100 \mathrm{ps}$ & $10 \mathrm{us}$ & $1 \mathrm{us}$ & $1 \mathrm{us}$ & $1.16 \mu \mathrm{s}$ \\
\hline $\boldsymbol{T}_{\boldsymbol{R}}$ & $0.54 \mathrm{~ns}$ & $15 \mathrm{us}$ & $200 \mathrm{~ns}$ & $39 \mathrm{~ns}$ & $160 \mathrm{mV}$ \\
\hline Undershoot & $90 \mathrm{mV}$ & $60 \mathrm{mV}$ & $16 \mathrm{mV}$ & $140 \mathrm{mV}$ & $160.281 \mathrm{~ns}$ \\
\hline FOM & $0.032 \mathrm{~ns}$ & $11.2 \mathrm{~ns}$ & $0.132 \mathrm{~ns}$ & $0.077 \mathrm{~ns}$ & 2.28 \\
\hline Decoupling & $0.6 \mathrm{nF}$ & - & - & - & - \\
\hline
\end{tabular}

fast a system can be made with a certain current efficiency. The smaller the FOM, the better the regulator.

$$
F O M=T_{R} \frac{I_{q u i e s c e n t}}{I_{L, \max }}
$$

The chip area consumed by this design is considerably smaller than [3] and comparable with [1]. Assuming the layout was optimized and matched the performance on schematic level, the results of this work show a promising performance in terms of FOM compared to [4] and [3]. This topology can also be designed to drive greater capacitive loads which can be achieved by increasing the current in $Q_{1}$ for a higher $g_{m 1}$.

\section{CONCLUSiON}

A new capacitor-free linear voltage regulator utilizing multi-loop control, suited for small system-on-chip applications, was designed. With its fast transient performance it demonstrated results comparable to or better than other similar designs from the literature. Simulation results showed that an undershoot of $140 \mathrm{mV}$ with a rise time of $39 \mathrm{~ns}$ occured when a $1 \mu \mathrm{s}$ load transient variation from $0-50 \mathrm{~mA}$ was applied.

\section{REFERENCES}

[1] P. Hazucha, T. Karnik, B. Bloechel, C. Parsons, D. Finan, and S. Borkar, "Area-efficient linear regulator with ultra-fast load regulation," IEEE J. Solid-State Circuits, vol. 40(4), pp. 933-940, 2005.

[2] "Selecting LDO regulators for cellphone designs," Maxim, 2001, application note 898 .

[3] T. Jackum, G. Maderbacher, W. Pribyl, and R. Riederer, "Fast transient response capacitor-free linear voltage regulator in $65 \mathrm{~nm} \mathrm{cmos}$," in Proceedings - IEEE International Symposium on Circuits and Systems, 2011, pp. 905-908.

[4] M. Loikkanen and J. Kostamovaara, "A capacitor-free cmos low-dropout regulator," in Proceedings - IEEE International Symposium on Circuits and Systems, 2007, pp. 1915-1918.

[5] X. Tang and L. He, "Capacitor-free, fast transient response cmos lowdropout regulator with multiple-loop control," in Proceedings of International Conference on Asic, 2011, pp. 104-107. 